\title{
Exploratory Study on Exercise Induced- Plasmatic Follistatin Kinetics: Proof of Concept
}

\author{
Valeria Pérez del Toro-Cuaik ${ }^{1 \#}$, Jessica Saba-Behar ${ }^{1 \#}$, Paola Domínguez-Oropeza ${ }^{2}$, Álvaro \\ Antonio Martínez-Saleh ${ }^{2}$, Israel Ramírez-Sánchez ${ }^{3}$, Guillermo Ceballos-Reyes ${ }^{3}$ and Gabriela \\ Gutiérrez-Salmeán ${ }^{1 *}$ \\ ${ }^{1}$ Facultad de Ciencias de la Salud, Universidad Anáhuac México (Campus Norte) \\ ${ }^{2}$ Escuela de Ciencias del Deporte, Universidad Anáhuac México (Campus Norte) \\ ${ }^{3}$ Laboratorio de Investigación Integral Cardiometabólica. Escuela Superior de Medicina, Instituto Politécnico
}

Nacional

\begin{abstract}
Received: : November 18, 2016; Accepted: December 06, 2016; Published: December 08, 2016
*Corresponding author: Gabriela Gutiérrez-Salmeán, Facultad de Ciencias de la Salud. Universidad Anáhuac México (Campus Norte), Av. Universidad Anáhuac \#46. Lomas Anáhuac, Huixquilucan, Estado de México. 52786. México, Tel: [52][55] 5627 0210, Ext. 7230; E-mail: gabrielasalmean@yahoo.com, gabriela.gutierrez@anahuac.mx
\end{abstract}

\begin{abstract}
Background and objective: Follistatin is a protein that has been positively associated with increased myogenesis and decreased fat accumulation hence may be a potential target for preventing/treating obesity. Its expression is induced by exercise -which may, partly, explain the anti-obesity underlying metabolic pathways of physical activity-; however, not many studies have described its kinetics after performing validated exercise protocols that simultaneously evaluate the aerobic capacity. Thus we aimed to determine the plasmatic levels of exercise induced-follistatin and to correlate such with aerobic capacity.
\end{abstract}

Materials and methods: After performing a treadmill stress test (Bruce protocol; with 3-minutes incremental velocity and inclination, until fatigue or reaching $85 \%$ maximal cardiac frequency), blood samples were drawn (at 1,2, and 4 hours post-exercise) in order to asses follistatin kinetics. Results were correlated with maximum oxygen volume, calculated from protocol-validated equations.

Results: 5 moderately active women volunteers were included (aged: $21 \pm 0.7$ years, weight: $54.7 \pm 10.5 \mathrm{~kg}, \mathrm{VO}_{2}$ max: $57.4 \pm 16.1 \mathrm{~mL} /$ $\mathrm{kg} / \mathrm{min}$ ). After stress test, a progressive decrease in the expression of follistatin during the first 2 hours was observed $(\mathrm{m}=-892)$; levels were found again increased at 4 hours. A negative correlation between aerobic capacity and exercise-induced follistatin levels were found.

Conclusions: After exercise, follistatin levels exhibit a descending slope which, at 4 hours, again rises. The most relevant finding in this study was the negative correlation between aerobic capacity and follistatin expression, thus allowing the assumption that their metabolic and exercise-induced pathways may not be interrelated.

Keywords: Exercise; Follistatin; Aerobic capacity

\section{Background}

Follistatin is an autocrine glycoprotein whose main role refers to the antagonism and inhibition of molecules belonging to the super family of Transforming Growth Factor Beta (TGF- $\beta$ ), whose members are, in turn, involved in processes of cell proliferation and differentiation, inflammation and immunity [1].

Traditionally, follistatin has been studied as part of the pituitary-gonadal axis as it occurs within the anterior pituitary folliculostellate cells and, after secretion, follistatin has been shown to inhibit the synthesis of Follicle Stimulating Hormone (FSH). Complementarily, follistatin binds to -thus inhibitsactivin; hence it stimulates gonadotropic function, not in terms of uncontrolled cell proliferation but instead, enhances cell differentiation [2].

The study of follistatin has gained increasing interest because of the role of the hormone in myogenesis: it has been shown to antagonize the function of myostatin -member of the aforementioned TGF- $\beta$ - both animal models and humans $[3,4]$. This phenomenon results in a significant increase in muscle mass -as myostatin is a negative regulator of muscle growth potent because it activates activin, hence inhibits protein transcriptionand muscle strength [5] which, in turn, may be reflected in athletic performance [6]. Moreover, in recent years, the relationship between muscle mass and obesity has been widely described: in brief, muscle metabolism plays a key role in the prevention and chronic diseases associated to excess fat body mass (i.e., obesity).

The previous are only a few reasons why skeletal muscle is no longer seen as merely responsible for the movement, but is now to be considered a highly active endocrine organ which, in fact, synthesizes and releases substances -called myokines- into the circulation after various stimuli produced by exercise and whose action can be both para and endocrine. Within said myokines is follistatin. Interestingly, some studies have reported that gene expression of this protein is regulated by exercise and muscle stretching; in fact, it has been shown that expression of follistatin increases after the completion of a single session of exercise; however, the specific molecular mechanisms have not yet been 
clarified (although it has been suggested that the expression is rather hepatic vs. muscular-, which could be linked to the depletion of macronutrients that occurs during exercise) nor described the duration of such increase once the physical activity.

The objective of the present study was to therefore determine the plasmatic levels of exercise induced-follistatin during the recovery period and to correlate such with aerobic capacity.

\section{Materials and methods}

We conducted a proof of concept, including volunteer healthy young adults (20 - 25 years old), with normal weight (body mass index $>18 \mathrm{y}<25 \mathrm{~kg} / \mathrm{m}^{2}$ ), who performed moderately intense physical exercise regularly and signed the corresponding informed consent. Anthropometric assessment (weight and height) was performed according to Lohman's technique. Maximum Cardiac Frequency (MCF) and its correspondent 85\% were calculated $(\mathrm{MCF}=220$ - age) in attention to the exercise protocol, as described in the following section.

\section{Treadmill stress test}

Bruce's protocol was used; in brief, this test progressively increases both velocity and inclination every 3 minutes (starting at 1.7 miles/ hour; $10 \%$ elevation) in 7 stages (reaching up to 6 miles/ hour; $22 \%$ elevation). The test finalizes whenever: a) total ergometry time (21 minutes) ends, b) subject refers "maximum fatigue" and "physical inability to continue the stress test" or, c) subject reaches his/ hers $85 \%$ of MCF. Whichever the case, total test time was recorded and further used to calculate aerobic capacity (i.e., $\mathrm{VO}_{2} \max$ ) with the following formula:

$\mathrm{VO}_{2} \max (\mathrm{mL} / \mathrm{kg} / \mathrm{min})=4.38 \times($ time in minutes $)-3.9$

In addition, Heart Rate (HR) is also recorded immediately after finishing the stress protocol, 1 and 3 minutes afterwards.

\section{Follistatin plasmatic levels}

Before (i.e., prior to treadmill test), 1, 2, and 4 hours after completing stress test, venous blood samples were collected into plastic tubes containing citrate as anticoagulant. These were immediately centrifuged (3500 r.p.m., 15 minutes) and plasma was further separated into cryotubes. Follistatin determination was performed using an commercial immunocolorimetric kit $($ Abcam $®)$.

\section{Ethics}

The present study complies with the ethical principles stipulated in Helsinki Declaration, the Nuremberg Code, and Mexican regulatory laws (Ley General de Salud en Materia de Investigación). In accordance to the latter, this research implies a "minimum risk" as procedures considered as "routinely" (weight measurement, stress test, blood collection $<450 \mathrm{~mL}$ ) are employed. Moreover, participants were informed of all procedures prior to any intervention and, finally, they could withdraw consent whenever they want to.

\section{Statistical analysis}

Quantitative variables are presented as mean \pm standard deviation, unless otherwise stated; qualitative variables are presented as frequencies. For inferential analysis, repeated measures ANOVA with Bonferroni post hoc test were performed; correlations were evaluated according to Pearson. A statistical significance level of $\mathrm{p}<0.05$ was considered. All analysis was performed with GraphPad Prism $®$, v.5.

After an open call, 5 subjects -all females, players of the soccer team- were recruited from a local academic institution (Universidad Anáhuac México, Campus Norte). Demographic and anthropometric data are show in Table 1.

Total test time was $14 \pm 3.7$ minutes, yielding an overall aerobic capacity of $57.4 \pm 16.0 \mathrm{~mL} / \mathrm{min} / \mathrm{kg}$. All but one of the participants reached their corresponding 85\% of MHR (161.6 $\pm 21.0 \mathrm{bpm}$ ) thus stress test was ended. 3 minutes post-test, finishing heart rate decreased, in average, almost 50\% (83.0 \pm $18.9 \mathrm{bpm}$ ) with a referred level of fatigue of $5 \pm 1.7$.

Plasmatic follistatin kinetics is shown in Figure 1. No significant differences were found among test times, although a decreasing slope $\left(\mathrm{m}=-892.0 \pm 259.8, \mathrm{r}^{2}=0.92\right)$ presented during the first two hours of recovery, whereas a perfectly fit increased gradient $\left(m=970.0 \pm 948, r^{2}=1.0\right)$ was found in the late recovery (2-4 hours post-exercise).

Correlation analyses yielded a non significant correlation between between aerobic capacity and percent change in heart rate from test finishing $\left(r^{2}=0.51\right)$, as shown in Figure 2. Finally, aerobic capacity was negatively correlated $\left(\mathrm{m}=-273.6 \pm 66.5, \mathrm{r}^{2}\right.$ $=0.85, p<0.05$ ) with basal plasmatic follistatin (i.e., before stress test $)$, but shown a positive $\left(\mathrm{m}=1.5 \pm 0.7, \mathrm{r}^{2}=0.65, n s\right)$ correlation

\begin{tabular}{|c|c|}
\hline Variable & Mean \pm SD \\
\hline Age (years) & $21 \pm 0.7$ \\
\hline Weight (kg) & $54.7 \pm 10.5$ \\
\hline Maximum Heart Rate (bpm) & $199 \pm 0.7$ \\
\hline 85\% MHR (bpm) & $169 \pm 0.7$ \\
\hline
\end{tabular}

BPM: Beats Per Minute; MHR: Maximum Heart Rate; SD: Standard Deviation

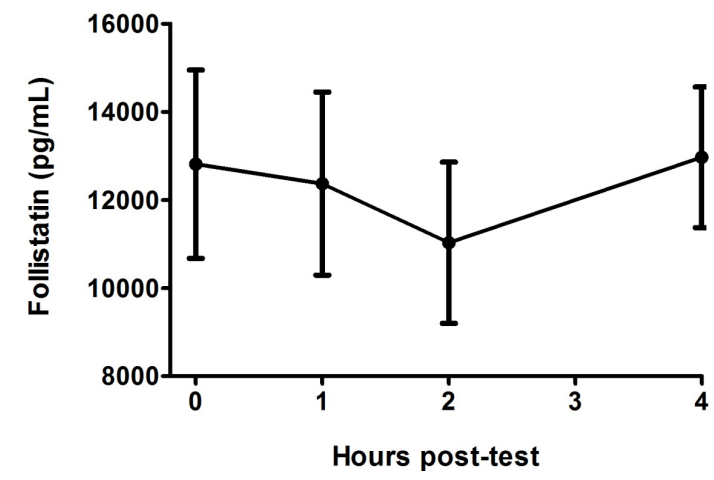

Figure 1 
to percent change (basal vs. 4 hours post-test) regarding follistatin blood levels (Figure 3, A and B, respectively).

\section{Discussion And Concluding Remarks}

Although not the initial aim of the present study, one of the main findings was that even though all volunteers were part of the University soccer team hence claimed to perform a moderatehigh level of physical activity (i.e., 2 hours of practice, 5 days a week), surprisingly, not one of the were able to conclude the stress test as they all reached $85 \%$ of MCF before completing 21 minutes of the Bruce protocol. But even more, standard deviations showed that results were greatly disperse among the recruited participants. This observation may be attributed to the fact that cardio-respiratory fitness and heart rate recovery have been associated with the in-field playing position of the study
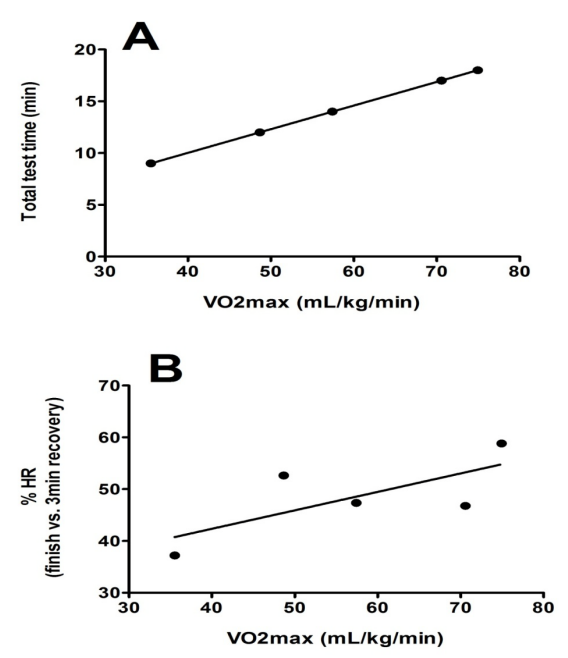

Figure 2
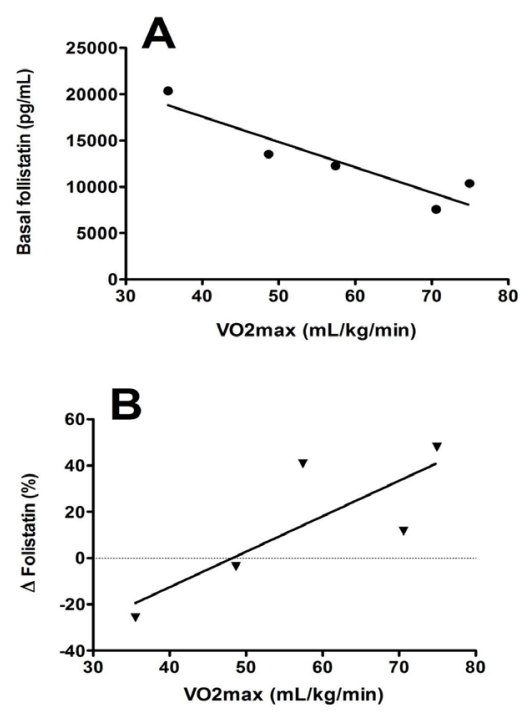

Figure 3 subjects [7], a confounding variable we did not take into account.

In this same thought, follistatin levels did not significantly differ from one time to another. This may be due to: a) small sample size and, b) wide variation that may be attributed to the previously mentioned basal cardio-respiratory fitness of each soccer player. Nevertheless, we found an ascending trend in follistatin plasmatic levels 4 hours after finishing the stress test. As ours, other studies have reported that the hormone's levels peak at 3 hours of recovery post acute exercise bouts [8].

Finally, even though a positive strong and significant correlation between $\mathrm{VO}_{2}$ max and exercise was certainly expected, the negative correlation with the prior with basal follistatin was not. This is not widely reported within the literature, although a study reported no significant changes in blood follistatin after resistance exercise and, moreover, authors attribute such to the fact that levels of plasmatic myokines are enhanced by moderate intensity exercise, rather than by resistance (i.e., high intensity) training [9]. This, in turn, may correlate with the types of muscle fibers (I or II): follistatin has been associated with type II (i.e., aerobic or slow twitching) fibers [10] thus may explain the aforementioned association with moderate (aerobic) exercise.

\section{References}

1. Ou Y, Zheng S, Lin L, Jiang Q, Yang X. Protective effect of C-phycocyanin against carbon tetrachloride-induced hepatocyte damage in vitro and in vivo. Chem Biol Interact. 2010;185(2):94-100. doi: 10.1016/j. cbi.2010.03.013.

2. Thompson TB, Lerch TF, Cook RW, Woodruff TK, Jardetzkyet TS. The structure of the follistatin: Activin complex reveals antagonism of both type I and type II receptor binding. Dev Cell. 2005;9(4):535-43.

3. Gutierrez-Salmean G, Ciaraldi TP, Nogueira L, Barboza J, Taub PR, Hogan MC, et al. Effects of (-)-epicatechin on molecular modulators of skeletal muscle growth and differentiation. J Nutr Biochem. 2014;25(1):91-4. doi: 10.1016/j.jnutbio.2013.09.007.

4. Amthor H, Nicholas G, McKinnell I, Kemp CF, Sharma M, Kambadur $\mathrm{R}$, et al. Follistatin complexes Myostatin and antagonises Myostatinmediated inhibition of myogenesis. Dev Biol. 2004;270(1):19-30.

5. Lee SJ, McPherron, C. Regulation of myostatin activity and muscle growth. Proc Natl Acad Sci USA. 2001;98(16):9306-11.

6. Nogueira L, Ramirez-Sanchez I, Perkins GA, Murphy A, Taub PR, Ceballos G, et al. (-)-Epicatechin enhances fatigue resistance and oxidative capacity in mouse muscle. J Physiol. 2011;589(Pt 18):461531. doi: 10.1113/jphysiol.2011.209924.

7. Bonaiuto M, Di Mauro D, Speciale F, Pagano F, Buda D, Vita G, et al. Evaluation of heart rate recovery in relation to playing position in professional soccer players. J Sports Med Phys Fitness. 2012;52(6):583-8.

8. Jakob Hansen, Claus Brandt, Anders R. Nielsen, Pernille Hojman, Martin Whitham, et al. Exercise induces a marked increase in plasma follistatin: evidence that follistatin is a contraction-induced hepatokine. Endocrinology. 2011;152(1):164-71.

9. Yeo NH, Woo J, Shin KO, Park JY, Kang S. The effects of different exercise intensity on myokine and angiogenesis factors. J Sports Med Phys Fitness. 2012;52(4):448-54.

10. Hosoyama T, Yamanouchi K, Nishihara M. Role of serum myostatin during the lactation period. J Reprod Dev. 2006;52(4):469-78. 